\title{
Development of a metamodel to foster interoperability along the product lifecycle traceability
}

\author{
Sergio Terzi ${ }^{1,2}$, Jacopo Cassina ${ }^{1}$, Hervé Panetto ${ }^{2}$, \\ ${ }^{1}$ Politecnico di Milano, Department of Economics, Management and Industrial Enginee- \\ ring, Piazza Leonardo da Vinci, 32, \\ 20133, Milano, Italy \\ sergio.terzi@polimi.it \\ ${ }^{2}$ Centre de Recherche en Automatique de Nancy, CRAN CNRS UMR 7039, \\ BP239 - F54506 Vandoeuvre-les-Nancy - France \\ Herve.Panetto@cran.uhp-nancy.fr
}

\begin{abstract}
The paper summarizes the effort spend by the authors in developing a model enabling the interoperability of systems for managing the product traceability along the entire product lifecycle. The research effort adopts an holonic definition of the traceability problem and, analysing the current most important standard in product representation, proposes a reference data model.
\end{abstract}

\section{Introduction}

Within the actual competitive world, enterprises are ever more stressed and subjected to high market requests. Customers are becoming more and more pretentious in terms of products quality and related services. The best product, at the low price, at the right time and into the right place is the only key success for the modern enterprise. In order to maintain or gain competitive advantages, modern enterprise has to manage itself along two main directions:

1. Improve internal and external efficiency, reducing all the not-relevant costs;

2. Improve innovation: innovation of product, process, structure, organization.

According to these needs, enterprises have to focus on their core-competences in order to improve the gained efficiencies (managing innovation) and to reduce the inefficiencies. Looking to this research, the product is re-becoming, after the soapbubble new-economy experiences, the real enterprise value creator and the whole production process is re-discovering its role. By this way, within the globally scaled scenario, product and production management have been become complex processes where more problems are overlapping each others. Product development might ever more take into account improved customers' tastes and requests in a shorter time-tomarket. The related engineering activities are consequently stressed, while inefficiencies in the production and distribution functions not are ever tolerated. 
This way, the product and production lifecycle and its related management are becoming unavoidable key aspects, creating such a "product centric" or "product-drien" problem. The integrated management of all the information regarding the "product" and its production is one of the related questions.

\subsection{Product Lifecycle Management}

One of the answers to these questions is already on going and could be advocated as a new emerging paradigm, defined as Product Life Cycle Management (PLM). In fact, listening to the enterprise questions, several vendors, coming from the diverse worlds interested into the product and production management, are more and more providing answers, stabling a growing tagged PLM market. Looking to this market, it is clear as a variety of "solution-providers" aim to be considered:

1. Vendors coming from the digital engineering world (UGS PLM Solutions, Tecnomatix, IBM-Dassault), which start from PD (Product Development) and MSE (Manufacturing System Engineering) processes and are trying to connect Enterprise Engineering and Management processes;

2. Vendors coming from the ERP world (Baan, SAP, Oracle), which, at the contrary, start from Enterprise Management processes for turning to connect PD/MSE tools and platforms;

3. Vendors coming from the ICT world, dealing software with architecture and platforms, which aim to establish such collaborative environments for PLM integration (Microsoft, MatrixOne, Agile), basically using web technologies.

It is important to notice that the needed product and production management is intrinsically related to the management of the information, so it is obvious that the related emerging market is IT characterized. Nevertheless, PLM is not primary an IT problem, but at first, is a strategic business orientation of the enterprise ([1],[2]). From a strategic organization point of view, the adoption or a product and production centric approach signifies a (re-)modelling of the all relations established between the resources (people and equipment) involved into the relevant business processes oriented to a "product" lifecycle directions, with all that it concerns in terms of task allocations and measurement of the obtainable performances. From the ICT point of view, a centric product and production management is no more than a "database" problem, which physically enables the previous business process modelling. Information about products and processes are dispersed along a variety of information systems, which - until now - has been executed no more than "isolated islands" (e.g. PDM (product Data management) and ERP). The trend and issue currently on going deal with the integration of these "islands" into a larger integrated (even if distributed) repository, in order to provide a wider and more effective use of product and production information. From a structural point of view, the instantiation of a product and production centric management approach signifies the product centric design and management of several elements:

1. An information infrastructure, which concerns with IT network establishment; 
2. A resource infrastructure, which concerns with the design and the management of all physical elements involved along a product and production lifecycle (e.g. machines, plants, people, suppliers, warehouses...);

3. A product itself "infrastructure" where the same product has become a resource to be managed directly, traced into its same lifecycle.

\subsection{Interoperability dimensions in the Product Lifecycle Management}

The main problem of this "PLM world" is the interoperability dimension: interoperability in terms of product data at ICT levels, but also interoperability in terms of persons, resource and systems that might cooperate and be in contact. The dimension of interoperability with a product-centric point of view becomes the problem of managing the tracing of the product along its lifecycle, or in other words, a problem of traceability.

This paper aims to discuss the problem of traceability as a problem of interoperability; in particular the paper aims to present the development of a reference metamodel for product traceability in a PLM wider scenario. The paper shows the preliminary results of a common effort, conducted by Politecnico di Milano and CRAN (Centre de Recherche en Automatique de Nancy), for providing an effective and reliable model to trace product and foster interoperability in the whole product lifecycle.

The paper is organized as follows:

- The second paragraph will present the definition of traceability in the lifecycle and will introduce an innovative point of view (based on holons) as a solution for product traceability and PLM interoperability.

- The third paragraph will illustrate the development of the proposed metamodel, realized on a reference model of product lifecycle considering the requirements of a potential user of this model, and taking into account the current status of interoperability in PLM, searched in Enterprise Standards.

- The fourth paragraph will will conclude the paper.

\section{Product Traceability}

The term "traceability" related to the product or manufacturing has been defined since the 90ies [3], when a series of industrial needs had been highlighted into the establishment of ISO 9000 procedures. Generally, product traceability is the ability of a user (manufacturer, supplier, vendor...) to trace a product through its processing procedures, in a forward and/or backward direction [4]. Physically, the product traceability deals with maintaining records of all materials and parts along a defined lifecycle (e.g. from raw material purchasing to finished goods selling) using a coding identification.

Traceability systems are adopted, according to laws, in the food sector, in manufacturing, in the pharmaceutical sector, in distribution, in constructions. Traceability systems can be useful to increase quality and safety of the product, for brand protection, and can increase efficiency in production and distribution. 
Traceability has different meaning in literature: Internal Traceability, that is the traceability inside the factory and the production system and External that follows the product into his relations with customers, maintainers, suppliers, etc. [3]. Another meaning is between Backward and Forward Traceability [4] (Figure 1). Backward Traceability records information and data on the past history of the product. Forward traceability explains what will happen to a certain product, all the processes and output that the product in question went into. These information are written before the product production begins and aims to give all the information that are needed to the production. This kind of traceability could be very useful in automated manufactures[5].

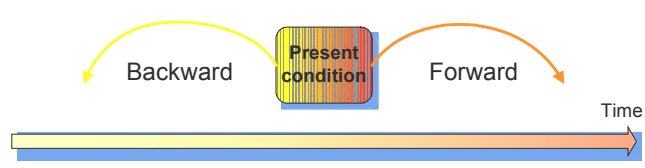

Figure 1. Backward and Forward traceability.

\subsection{Towards a Holonic Traceability}

At the present, the product traceability problem concerns with the identification of a product, even if often is only the type of product, using a coding system (e.g. bar code, laser code, EPC code [5]). All the information related to the coded "product" are then stored into one (or more) database. Therefore, a merging activity between the product and its information is an obligatory step, also in the most advanced issues (e.g. Auto-Id efforts in [5], or Dialog effort in [7]). This re-merging activity is still not risk-free; even if it's could be already conducted in an automated manner (e.g. [5], [6]), transactions breakdowns could occur [7] in searching for information into the database. In general, two main problems could be advocated:

1. Accessibility. Database could be off-line or unavailable for a short or long period.

2. Timing and Costing. Database could become too large and so expensive (or many database could be needed), moreover reducing efficient reading time.

A solving attitude could be identified in the concept partly illustrated in [8], where a simple 2D bar-code attached to physical elements had been adopted to translate highdensity information (whole plant drawings) from the plant designer to the contractor. Taking into account this example, each product could be provided with an advanced "product information store system" (e.g. RFID based), in order to be (i) from one side tracked into a system (e.g. a plant) and, from another side, (ii) to be able to provide itself the needed information. In such a vision, the product itself become the medium of the data set, instantiating a kind of "intelligent product" ([7], [9]), able to interoperate in the environment, exchanging information (which are into the product itself) in real-time with different resources (e.g. machines and transporters into a plant scenario, or trucks and inventory database into a warehouse, or with refrigerators and dishwasher at home...). 
Looking to the literature, the paradigm of "product + information" had been already developed and it is defined as holonic worldview. The word Holon was introduced by Koestler in 1967 [10], as a combination of the Greek Holos (whole) with the suffix on, which as in proton and neutron suggests a particle or individual part. In the 1993, the holonic term was applied to the manufacturing world, creating the Holonic Manufacturing Systems (HMS) community. For this community a Holonic Manufacturing "is an autonomous and co-operative building block of a system fro transforming, transporting, storing and/or validating information and physical objects. The holon consists of an information processing part and often a physical processing part. An holon can be part of another holon." [11].

A holonic-based product traceability could be a killer application into a PLM context. Lot of improvement could be gained establishing an intelligent product, sensibly reducing inefficiency in different processes, from the manufacturing one, to the distribution, to the after sales, to the quality assessment, till product accounting and selling.

\section{Development of a metamodel for product lifecycle traceability}

Looking to the Holonic Product Traceability research effort and thinking to the future, in some years a product holon could be inserted in more systems (e.g. a plant, a supply chain, a warehouse) where it will have to exchange information with different resource holons ([5], [9]). Hence, the problem of information exchange could easily arise and further standardization efforts will be needed, so establishing a kind of barriers to the diffusion of the same holonic traceability.

In order to reduce these further barriers, but ever more in order to improve the currently definition and the study of Holonic Product Traceability, a research effort could be spent since now, looking to the actual situation of enterprise information systems (where product information are resident) and try to elaborate it in an holonic view, creating a conceptual HMS product-oriented architecture.

The current situation of the enterprise information systems could be registered in the analysis of the current accepted standard, which are specifically created fro the integration of IT systems. The analysis of standard is a basic step that could reduce the research effort, avoiding a long state of the art analysis of enterprise IT systems.

\subsection{Product Lifecycle phases}

Traceability is an ability needed along the whole product lifecycle. In order to develop a wider reference model, a definition of lifecycle is needed. In literature there are many different "life cycle" models; trying to merge diverse kind of Product Lifecycle model, it is possible to identify the following Product Lifecycle Model, which will be considered in the work. This model standardizes a product lifecycle composed by four different phases:

1 Product Development: it deals the developing phase of the product, starting from product design and ending, through process and plant design. Each of 
these four product development sub-phases usually starts from the requirements analysis (requested performances, costs, marketing strategies and so on) and proceeds with a first draft for ending with the detailed design.

2 Product Production: it comprises both production and distribution activities. Production phase may be very complex and often includes pre-production and prototyping, manufacturing, assembling, finishing, testing, packaging, etc. Distribution, on the other side, is related with product storage and delivery.

3 Product Use: this is the proper product life phase and represents all activities which take place during product use: they comprise product usage and consumption, maintenance and support.

4 Product Dismiss: in this last phase the product is destroyed, or rather disassembled and recycled.

\subsection{Analysis of Enterprise Standards}

One of basic requirements for the development of a product traceability model was that of achieving an easy understanding of such a model by people or organizations of different countries, languages and cultures. This suggests avoiding misunderstanding of concepts, ideas or definitions making use, whenever possible, of shared standards. The authors had conducted a literature survey on standards for manufacturing control, product design, product support and so on. It is a matter of fact that there are many standards, each of one focused on a specific area of the product lifecycle, but none including all the information needed in the whole lifecycle chain, as shown in the next figure.

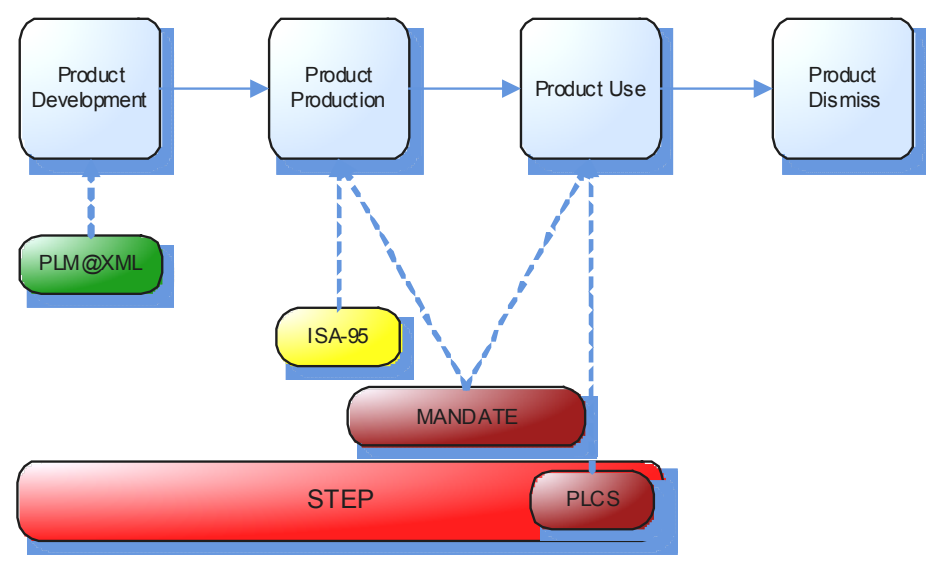

Figure. 2. Standards through Life Cycle Phases

Four standards or initiatives seem interesting to be studied because they are complementary in the PLM. They are ANSI/ISA-95 (or the new ISO/IEC 62264 standard which is derived from it), Mandate (ISO 15531), PLCS (ISO 103003-239), and PLM@XML. These standards share in common some properties and features, but are 
also distinguished by a lot of remarkable differences. First of all, they were designed by different organizations, with different scopes and for different targets. STEP, PLCS and Mandate can at first sight be grouped together, because each of them is an ISO (International Organization for Standardization) standard; furthermore, PLCS is an application protocol of STEP. STEP is an industry standard for product data representation and it's composed of several parts (application protocols) whose aim is to focus on a specific industrial context. There are application protocols for product design, for mechanical and electrical engineering, for sheet-metal manufacturing, for product assembly, for automotive industry and so on. PLM@XML is an open standard developed mainly by EDS (Electronic Data Systems Corporation) and it deals with the product design phase. ISA-95 is an ANSI (American National Standard Institution) standard, but its first part, ANSI/ISA-95.00.01, is also an ISO standard (ISO 62264-1). ANSI/ISA-95 Parts I, II, and III describe the interfaces and activities between an enterprise's business systems and its manufacturing control systems: it focuses, thus, mainly on the area corresponding to our product production phase.

Another interesting initiative is the Physical Markup Language (PML), under development at Auto-ID laboratories [5]. PML is intended to be a general, standard means for describing the physical world. The objective of PML is a simple, general language for describing physical objects for use in remote monitoring and control of the physical environment. PLM was thought as a part of a wider structure, built around four major components: electronic tags, Electronic Product Code (EPC), Physical Markup Language (PML) and Object Naming Service (ONS).

\subsection{Definition of the requirements of the model}

In order to develop a comprehensive model, the User Requirements and General Requirements had been developed analyzing the literature; the first are needs explicitly explained in literature by traceability users, the latter are implicit in literature and are useful for every possible user. The User Requirements are more specific for each single industrial sector, for example it exists specific requirements for pharmaceutical industries, others for the distribution etc. These are also specific for each life cycle phase, as showed in figure 3.

General requirements are needs implicitly written in literature and not clearly declared. Sometimes, in literature, authors are concerned with some matters of product traceability, especially when referring to a specific context of application. Product traceability is usually dealt by people with different cultural backgrounds, just because traceability is required - and thus studied - for agricultural uses as well as for manufacturing industry, for software development as well as for healthcare. This way it's possible to look at the problem concentrating only on a little part of it and losing a wide-spread view.

The defined General requirements are:

1. Product Descriptive Power: The model should be able to describe very different products.

2. Multi-Scenario Descriptive Power: In Literature there are many scenarios and many mono-scenario models, but a multi-scenario model is missing [12]. The model has not to fit a special scenario, industrial sector, context of appli- 
cation or environment, but shall fit each time any different context without needs for modifications.

3. Updatable: The model has to follow the evolution of the product, and keep track of information describing modifications and operations made on it. It's shall include information and data necessary for forward traceability and, at the same time, guarantee recording of product history (backward traceability).

4. Lifecycle scalability: The model should describe different phases of lifecycle, and should describe them in such a way to be useful for both single phase oriented users and whole chain lifecycle oriented user.

5. Detail scalability: The model should describe different detail level, comprising product as well as subcomponents.

6. Being distributable: The information should be stored in different supports (RF tags, barcodes, and database). Due to technological reasons, for example the amount of free memory on an RF tag for storing information, it's sometimes impossible to keep the whole description of product lifecycle together with the physical product itself; so the information has to be divided part on the product part on a remote storage.

7. Shareable: The information should be shared between many users and industries.

8. Trusted access: To grant information restraint to different kinds of users.

9. Unambiguously understandable: Many users of different cultures and languages have to access information; the model should avoid misunderstandings. For this purpose we suggest usage of standardized information and model, taking into account wide spread standards whenever possible.

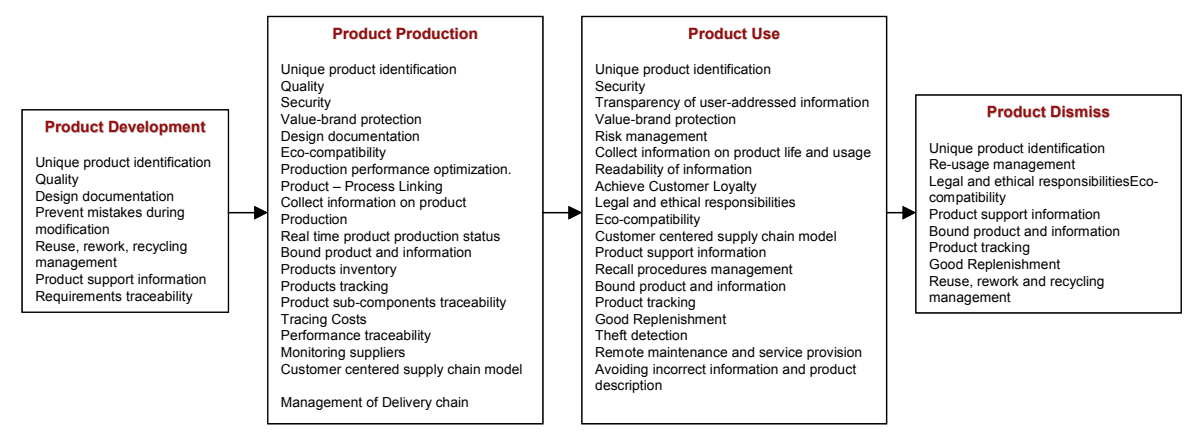

Figure 3. User Requirements in Life Cycle Phases.

\subsection{The Holonic Traceability Model}

Taking into account the previously presented requirements and the Holon concept defined in [13] (figure 4), the model for Holonic Product Traceability is hereafter defined. 
Figure 4 explains that the Holon results from the linking of a Physical Object and some information. There are many information related to the product and they are defined by the ObjectInformation class. If the link between the physical object and its related information is missing the idea of Holon vanishes and the traceability model miss its target. The link can be established using many technologies, barcodes and databases, RF Tags, etc, but this technological implementation is out of the aim of this work; in fact it is concentrated on the information needed to ensure traceability.

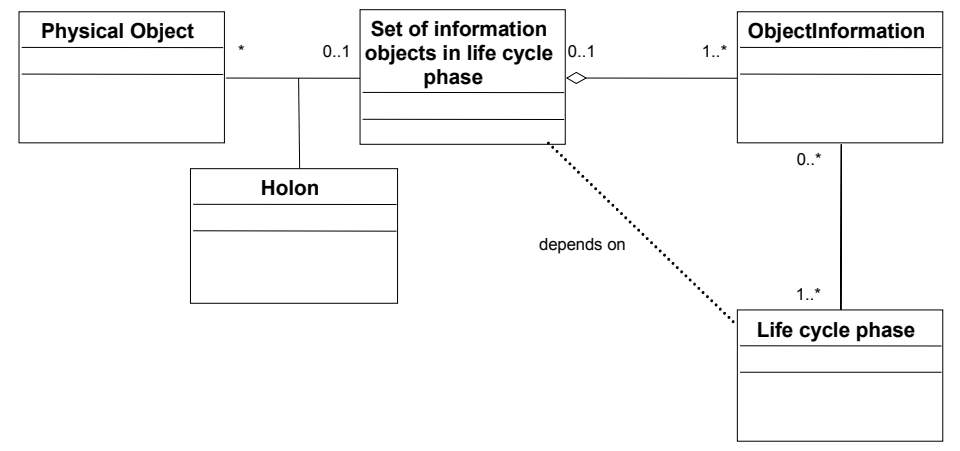

Figure 4. Definition of Holon (derived from [13])

The proposed model is mainly focused on defining the Information needed to ensure traceability (figure 5). ObjectInformation is a group of information that can summarize all the life of the product; it can follow the product during its lifecycle phases, like, for example, during its production, or use. The ObjectInformation class contains general information on the product as the identification, class and batch information, a description of the product, it's characteristics and the results of possible tests made on it. The main elements of this class were derived from ISA/ANSI 95 and ISO 10303 AP 239 (PLCS). 


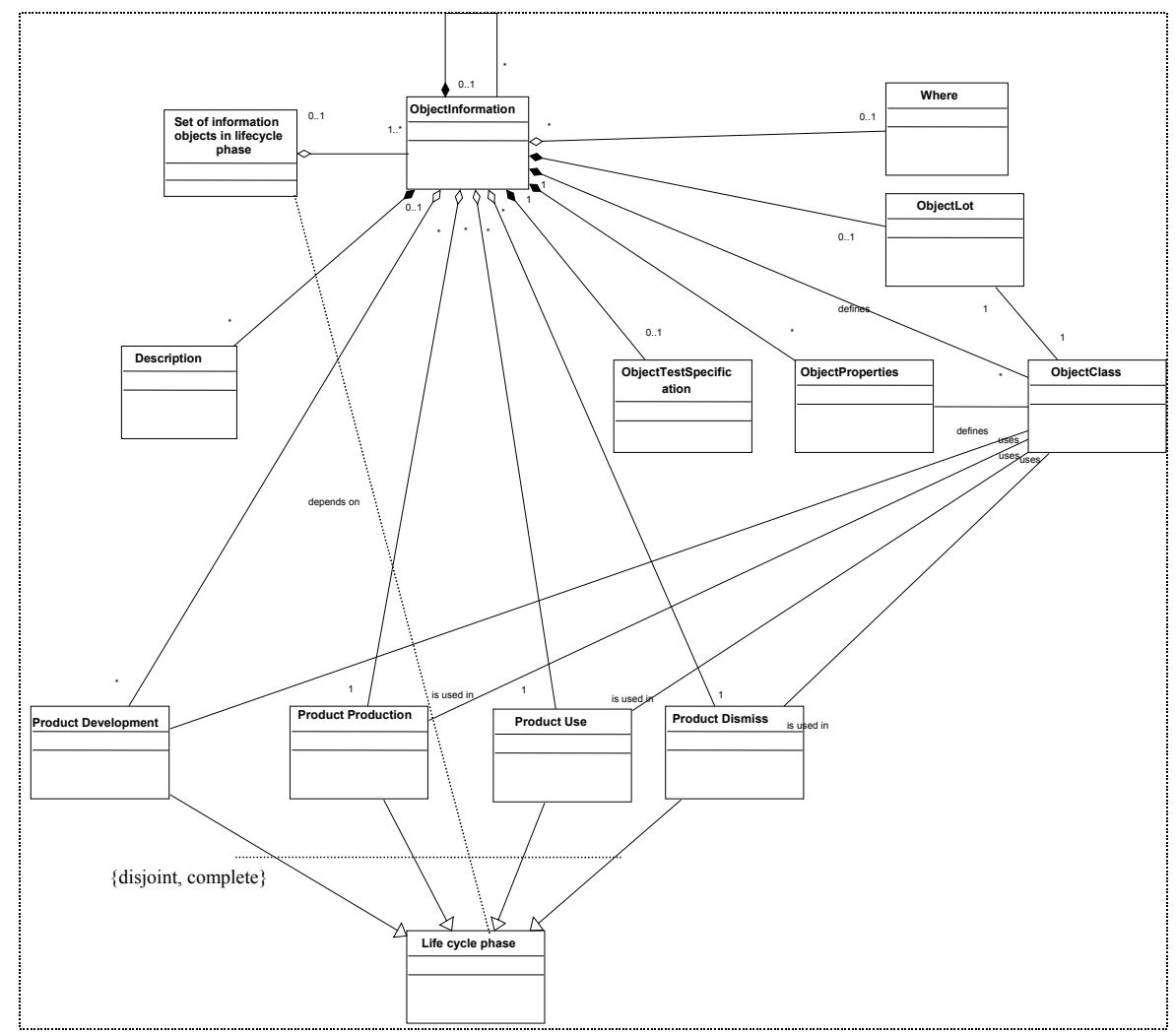

Figure 5. The Object Information schema

It also records information about the lifecycle of the product contained into four specialization of the Life Cycle Phase class (figure 6). This class describes a generic phase of the lifecycle, using the Event, Activity and Resource classes. An Event causes an Activity that uses Resources. The model can be used both for backward both for forward traceability; for the backward it'll use the EventAsOccurs class that records how and when the event occurs and ActivityAsRealized class that describes how the activity has been fulfilled. It is also possible to implement a Forward Traceability using the EventAsPlanned class that describes how and when an event has to occur and the ActivityAsPlanned that explains how to do it. When the event and the activity really occur they are recorded into the model as backward traceability, exploiting the EventAsOccurs and the ActivityAsRealized classes.

The model has a fractal structure. It is used recursively to describe the components of the tracked product. All the classes are more detailed; for example $I D$ is composed by an Objectld that identifies each istance of the physical product and a ONS (Object Naming Service) as proposed by Auto-Id [5]. 


\section{Conclusions}

With this work it has been proposed a innovative vision that is the Holonic approach, for the traceability as well as the management of life cycle data. This innovative approach aims to foster interoperability along the diverse enterprise applications, in particular at a manufacturing stage. We propose a meta-model supporting the informational part for the traceability of products (or Holons-products), along its life cycle. This model was established, re-using, at best, existing work around some standards: PLCS, Mandate, ANSI/ISA-95, PLM/XML and PML-EPC. The model is technology independent and fits to different application domains. The model can trace the history of the product in details, or can be less detailed, but more compact such a way all the information can be written on a $2 \mathrm{D}$ barcode. The model can also use a combination of barcode and remote databases; on the barcode could be written the most frequently used information; the remote database could record less exploited information. The model could also be implemented on RFID tags that could contain all the necessary information. The ongoing research particularly relates to the methodological aspects regarding the modeling process of enterprise and manufacturing processes, by taking into account, a priori, the products traceability objectives during the design stage and the integration phase of the productions systems, or when reengineering the existing ones. Also data management has to be deepened, especially the security and the privacy which is an mandatory for consumers. Finally, cost estimation on the implementation and the management of traceability systems is needed.

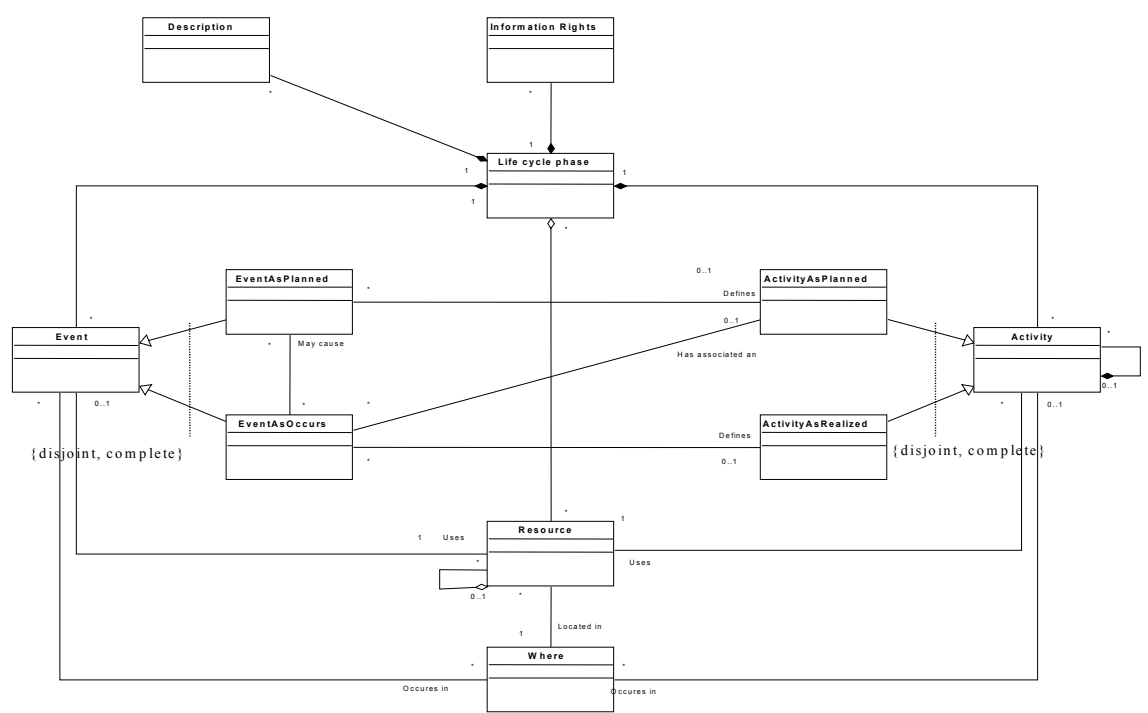

Figure 6. The Life Cycle Phase class 


\section{References}

[1] CimData, 2004, www.cimdata.org

[2] Garetti M. Terzi S., Product Lifecycle Management: definition, trends and open issues, Proceedings at III International Conference On Advances In Production Engineering, 17 19 June 2004, Warsaw, Poland

[3] Cheng M.L. and Simmons J. E. L., 1994, Traceability in manufacturing systems. International Journal of Operations and Production Management, 14, 4-16

[4] Jansen-Vullers J., A. van Dorp, B. Beulens, 2003, Managing traceability information in manufacture, 2003, International Journal Of Information Management $23: 395-413$

[5] McFarlane D., J. Sarma, G. Chirn, J. Wong, A. Ashton, 2003, Auto-ID systrems and intelligent manufacturing control, Journal of Engineering Applications of Artificial Intelligence, $16: 365-376$

[6] McFarlane D. J., Bussmann D., 2000, Developments in Holonic Production Planning and Control, Int. Journal of Production Planning and Control, Vol. 11, No. 6, pp. 522 - 536

[7] Kärkkäinen J., G. Holmström, J. Främling, G. Artto, 2003, Intelligent products - A step towards a more effective project delivery chain, Computers in Industry $50: 141-151$

[8] Finch F., M. Flanagan, M. Marsh, 1996, Electronic document management in construction using auto-ID, Automation in Construction, $5: 313-321$

[9] Morel G., H. Panetto, A. Zaremba, G. Mayer, 2004, Manufacturing enterprise control and management system engineering rationales and open issues, IFAC Annual Reviews in Control, $27: 199-209$

[10] Koestler A., 1967, The Ghost in the Machine, Arkana Books, London

[11] Seidel D., Mey M., 194, IMS - Holonic Manufacturing Systems: systems components of autonomous models and their distributed control - Vol 0. Also known as The Book of HMS, TC5 project

[12] Ramesh B., Stubbs C., Powers T., Edwards M., 1997, Requirements traceability: Theory and practice, Annals of software engineering

[13] Gouyon D., Simão J. M., Alkassem K., Morel G., 2004, Work in progress for product driven manufacturing automation, 2004, Proceedings of IFAC INCOM Symposium, April 7th-9th, Salvador de Bahia, Brasil 